\title{
IMPORTANCE OF SEROLOGICAL WORKUP IN CATARACT CASES OF OUT-REACH PROGRAMMES
}

H. N. Sowbhagya, Kiran Kumar L, Nivedhitha Nikhil, Kirthi Raj, Liji P, Pooja Patil, Aisha Rafi , Sushruth. A.

1. Professor. Department of Ophthalmology, Kempegowda Institute Of Medical Sciences And Research Centre.

2. Assistant Professor. Department of Ophthalmology, Kempegowda Institute Of Medical Sciences And Research Centre.

3. Post Graduate. Department of Ophthalmology, Kempegowda Institute Of Medical Sciences And Research Centre.

4. Post Graduate. Department of Ophthalmology, Kempegowda Institute Of Medical Sciences And Research Centre.

5. Post Graduate. Department of Ophthalmology, Kempegowda Institute Of Medical Sciences And Research Centre.

6. Post Graduate. Department of Ophthalmology, Kempegowda Institute Of Medical Sciences And Research Centre.

7. Post Graduate. Department of Ophthalmology, Kempegowda Institute Of Medical Sciences And Research Centre.

8. Post Graduate. Department of Ophthalmology, Kempegowda Institute Of Medical Sciences And Research Centre.

\section{CORRESPONDING AUTHOR:}

Dr H. N. Sowbhagya,

No 41-42/45 Sri Guru Krupa,

7th Cross, Saraswathipuram,

Nandini Lay Out Main Road,

Bangalore- 560096.

E-mail: drhnsowbhagyaappaji@gmail.com

ABSTRACT: PURPOSE: The aim of this study is to determine the frequency of positive serology among patients who underwent elective cataract surgery. METHODS: Retrospective study of 800 cases who underwent thorough laboratory work up as a part of clinical study. 490 were male $(61.25 \%)$ and $310(38.75 \%)$ were female. 35 cases $(4.37 \%)$ were positive for serology. Hepatitis A in 9(1.13\%) cases , Hepatitis B in $6(0.75 \%)$ cases ,hepatitis C in 10 (1.3\%)cases ,HIV positive in $8(1 \%)$ cases , VDRL in $2(0.25 \%)$ cases. All cases were grouped as high risk cases for Operating theatre (OT) supportive staffs and surgeons and operated with extra caution. RESULTS: Retrospective analysis of incidence of positive serology in camp cases were found significant. All were operated with all safety measures to protect surgeons and other supportive staffs. CONCLUSIONS: Study proves that serological work up is necessary for all the cases posted for elective cataract surgery. Serology positive patients need special attention to safe guard the surgeon and other supportive staffs

KEYWORDS: Elective Cataract surgery, serology, Non manifest sero positive cases, professional health hazard, protective measures.

INTRODUCTION: Cataract is a preventable cause of blindness and cataract surgery is the most common surgery performed worldwide to restore vision. As per NPCB2011 (National Program for Control of Blindness), $1 \%$ of the Indian population (121 core) is blind. Of this, $62 \%$ are blind due to cataract. Incidence of cataract is $0.4 \%$ to $0.5 \%$. Approximately 7 million people 
are/become blind due to cataract at any point of time. India is performing 6 million cataract surgeries every year ${ }^{1}$.

In our population, when patients report for poor vision due to senile cataract, they also suffer from other medical problems like diabetes, hypertension. Cataract Patients from outreach programs are not routinely screened for serology. Since majority of carriers are asymptomatic, they pose a real threat to health staff. Approximately 34.2 million people were living with HIV in 20112. A literature review revealed reports of 33 health care workers who had contracted HIV due to their occupation ${ }^{3}$. Hepatitis B and Hepatitis C are most common causes for viral Hepatitis leading to chronic infection and carrier state. These carriers are a major threat for the spread of the disease. Hepatitis B is endemic worldwide, responsible for 1-2 million deaths worldwide every year. About 2 billion people have current or past infection and 350 million (24\%) are carriers of the virus, out of which $80 \%$ reside in Asia. Indian subcontinent is classified as an intermediate HBV endemic (HBs Ag carriage 2-7\%) zone and has the second largest global pool of chronic HBV infection 4 . The infection leads to chronic carrier state in $60 \%$ of affected individuals 5 . Hepatitis C prevalence, according to WHO estimates is $3 \%$ of world population with 150 million chronic cases ${ }^{6}$. Most common risk factors for transmission of Hepatitis B, Hepatitis C, and HIV virus are per cutaneous exposure to blood, major surgeries, dental treatment, intravenous drug abuse, tattooing, use of contaminated syringe and blood transfusions. Surgeons and paramedical staff and other staff are at increased risk to get infected, especially in a surgical setup where unknown carriers of the virus are undergoing various procedures.

Risk of exposure during surgery in ophthalmology includes out-patient (OPD) procedures like syringing, biometry, Tonometry and OT procedures during anaesthesia, handling of sharp instruments (Blade, side-port, needles) and during cleaning and exchange of instruments, disposal of biomedical waste.

METHODS: Retrospective study of 800 cases who underwent thorough laboratory work up, chest X-ray and electrocardiogram as a part of study after obtaining IEC clearance and patients informed consent. Study done as per the guidelines of ICMR and WMA Declaration of Helsinki. Laboratory work-up included haematology, biochemistry and urine analysis and serology for Hepatitis A, Hepatitis B, Hepatitis C, HIV, and VDRL from May 2011 to February 2012. Serology work up was done by Electrochemiluminiscence to detect HIV I and HIV II antigen and antibody, antigen of hepatitis B and antibody against hepatitis C virus. RPR (Rapid plasma reagent) was done to detect syphilis antigen. Ethical considerations like confidentiality about the study patient's name and their results were maintained. All cases were grouped as high risk cases for OT supportive staffs and surgeons, operated with extra protocol, for all these high risk cases OPD procedures like syringing was done at the end of screening of other cases using disposable cannulas. Biometry was done using ultra thin transparent polythene paper interface and the tip disinfected. Tonometry done using rebound Tonometer and probes were discarded. OT procedures included use of disposable linen, double gloves to surgeon and assistants and use of disposable instruments. Sharp instruments were handled with care to avoid cuts. Reusable instruments like blade handles, scissors and others are identified as separate sets and cleaned with glutaraldehyde (Korsolex) for prescribed time, cleaned and then double autoclaved. Biomedical wastes were disposed as per the biomedical waste disposal protocols. 
RESULTS: A Total of 800 patients were screened, 490 were male (61.25\%) and 310(38.75\%) were female. Age ranged between 40 to 60years, males showed higher incidence. 35 cases $(4.37 \%)$ were positive for serology (Diagram 1$)$. Hepatitis A in $9(1.13 \%)$ cases , Hepatitis B in 6 $(0.75 \%)$ cases, hepatitis $\mathrm{C}$ in $10(1.3 \%)$ cases, HIV positive in $8(1 \%)$ cases, VDRL in 2 $(0.25 \%)$ cases. Out of the 35 cases, none of them were symptomatic of the diseases. Frequency of HBV ( 6 cases) was $83.3 \%$ (5) in males and $16.6 \%$ (1) in females. Frequency of HCV (10 cases) was $60 \%$ (6) in males and $40 \%$ (4) in females. Frequency of HIV (8 cases) was $62.5 \%$ (5) in males and $37.5 \%$ (3) in females. Frequency of VDRL ( 2 cases) was $50 \%$ (1) in males and $50 \%$ (1) in females. Frequency of hepatitis A (9 cases) was $55.5 \%$ (5) in males and $44.4 \%$ (4) in females (Diagram 2). The frequency of Hepatitis B, Hepatitis C and HIV (combined) was more in the age group ranging between 45-60 years in both sexes. All cases were grouped as high risk cases for OT supportive staffs and surgeons, operated with extra protocol.

Nearly 3-4\% cases were found to be positive for serology in this study. Hence, serology screening should be made mandatory to protect surgeons and other supportive staffs from risk of infection.

DISCUSSION: The sero-prevalence of HIV infection in our series was $1 \%$ with infection being more common in males as compared to females. The frequency of HBV was more in males than females while there was not much gender difference found as for as HCV was concerned. The greater frequency of HBV infection in males as compared to females could be a reflection of more social mobility in males than females and thus greater vulnerability to be infected. This finding is comparable to a number of studies. 7,8,9 Both hepatitis B and C are highly prevalent in the age group between 45-55 years. It could be due to study being conducted among cataract patients, majority of whom are of middle to old age. The results show that the rate of HCV infection is higher than HBV in this study, which is in line with other studies. ${ }^{10,11}$ Doctors in surgical practice are at high risk of acquiring blood borne diseases from the patients on whom they operate ${ }^{12}$. $80 \%$ of HBV and HCV positive patients had no past history of jaundice. None of the 800 patients were found vaccinated against HBV. Prevalence of antibodies to HCV in health workers in developing countries are 20 folds higher than health workers in the developed countries $^{13}$. HIV infected individuals have an increased risk of cataract surgery ${ }^{14}$

This study also explains

1) The importance of mass vaccination of the health workers for hepatitis $B^{15}$.

2) Importance of setting up of extra protocol for this health hazardous cases.

CONCLUSION: The high prevalence of HBsAg and anti-HCV in the eye patients presenting for cataract surgery provides evidence for routine preoperative screening of all patients for surgery. Need for mass immunization against hepatitis B, and awareness regarding hepatitis B and $\mathrm{C}$ should be promoted among doctors, paramedical staff and general public. Periodic screening is to be made mandatory to detect latent cases of seropositivity to reduce the occupational hazard of health care professionals.

\section{REFERENCES}

1. National programme for control of Blindness. Quality aspects of cataract operations 2011. Available from npcb.nic.in/writereaddata/mainlinkfile/File 243.pdf. 
2. WHO/UNICEF/UNAIDS (2011) "Global HIV/AIDS response: Epidemic update and health sector progress towards universal access".

3. Veeken H, Verbeek J, Houweling H, Cobelens F. Occcupational HIV infection and Health care workers in the tropics. Trop Doc. 1991; 21: 28-31.

4. Lone DS, Aman S, Aslam M. Prevalence of hepatitis C virus antibody in blood donors of Lahore. Biomedica 1999; 15: 103-7.

5. Hepatitis C Fact Sheet No. 164 Geneva, World Health Organisation, 2000.

6. Khan TS, Rizvi F. Hepatitis B seropositivity among Chronic Liver Disease patients in Hazara Division Pakistan. J Ayub Med Coll Abottabad Sep 2003; 15: 54-5.

7. Mashud I, Khan H, Khattak AM. Relative frequency of Hepatitis B and C viruses in patients with Hepatic Cirrhosis at DHQ Teaching Hospital DI Khan. J Ayub Med Coll Abottabad Mar 2004; 16: 32-4.

8. Frequency of hepatitis b and hepatitis c among cataract patients Iftikhar Ahmad, Sardar Bahadur Khan2, Hafeez-ur-Rehman3, Muhammad Hussain Khan1, Saeed Anwar1 Gomal Journal of Medical Sciences July-Dec 2006, Vol. 4, No. 2. 61.

9. Aziz S, Khanani R, Noorulain W, Rajper J. Frequency of hepatitis B and C in rural and periurban Sindh. J Pak Med Assoc. 2010 Oct; 60(10):853-7.

10. Naeem SS, Siddiqui EU, Kazi AN, Khan S, Abdullah FE, Adhi I. Prevalence of Hepatitis 'B' and Hepatitis 'C' among preoperative cataract patients in Karachi. BMC Res Notes. 2012 Sep 6;5:492.

11. Ahmad J, Taj AS et al. Frequency of hepatitis B and hepatitis $C$ in healthy blood donors of NWFP: a single center experience 2004 Vol 18 No 3: Page $343-352$.

12. Rehman FU, Haq NU et al. Risk of Hepatitis in Surgical Practice J Postgrad Med Inst Jun 2002; 16: 157-60.

13. Aziz S, Memon A, Tily HI, Rasheed K, Jehangir K, Quraishy MS. Prevalence of HIV, Hepatitis B and C amongst Health Workers of Civil Hospital Karachi. J Pak Med Assoc Mar 2002; 52: 92-4.

14. Rasmussen LD et al .Risk of cataract surgery in HIV-infected individuals: a Danish Nationwide Population-based cohort study. Clin Infect Dis. 2011 Dec;53(11):1156-63. CDC. Immunization of Health care personnel: Recommendations of the advisory committee

15. on Immunization practices (ACIP). MMWR, 2011; 60 (RR-7). 


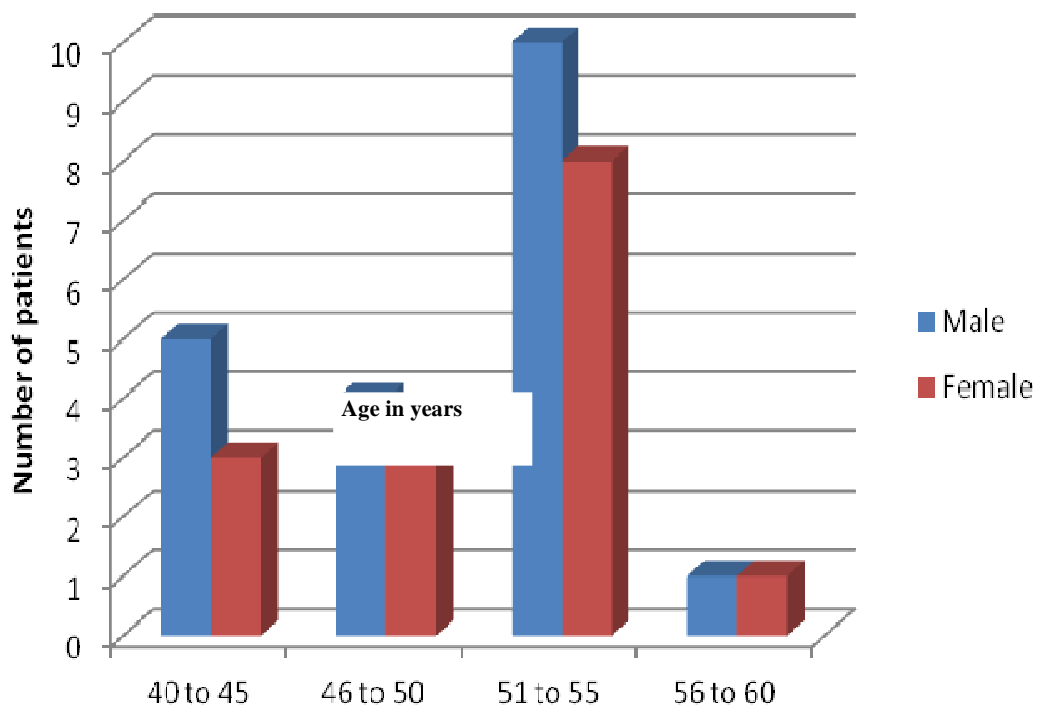

Diagram 1

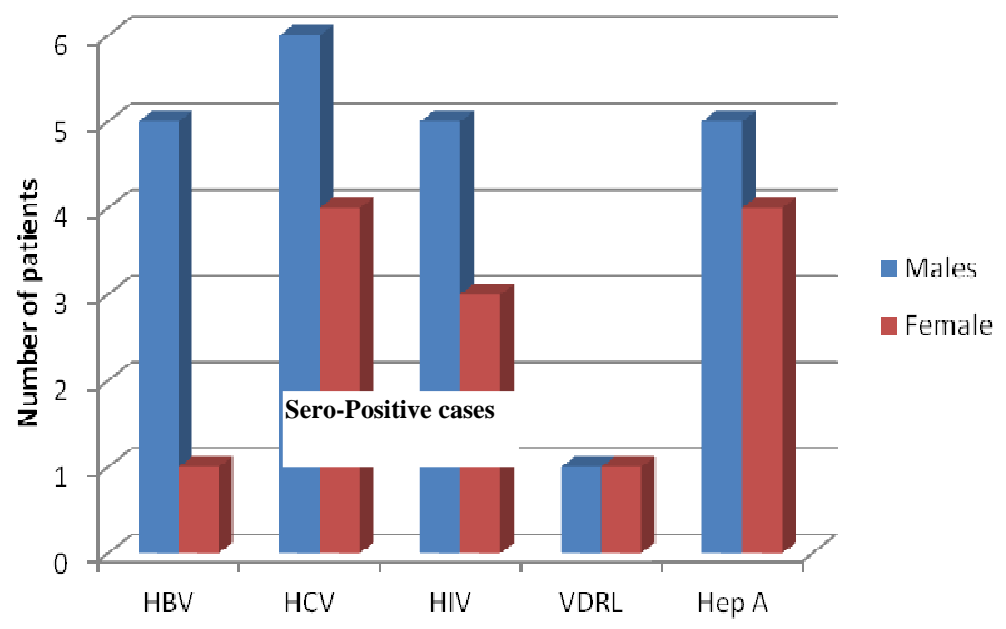

Diagram 2 